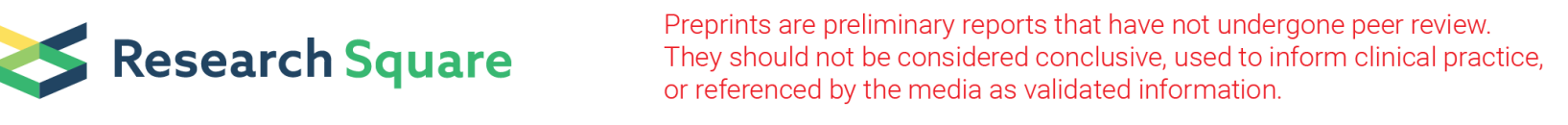

\title{
Direct Repair of Extensor Mechanism Disruption After Total Knee Arthroplasty "A Case Series of 31 Patients"
}

\section{Tiejian Li}

Chinese PLA General Hospital

Jing-yang Sun

Chinese PLA General Hospital

Yin-qiao Du

Chinese PLA General Hospital

Jun-min Shen

Chinese PLA General Hospital

Bo-han Zhang

Chinese PLA General Hospital

Yonggang Zhou ( $\nabla$ ygzhou301@163.com )

Chinese PLA General Hospital https://orcid.org/0000-0002-7304-4691

Research article

Keywords: Direct repair, Extensor mechanism disruption, Total knee arthroplasty, Reconstruction

Posted Date: August 30th, 2021

DOl: https://doi.org/10.21203/rs.3.rs-796317/v1

License: (1) This work is licensed under a Creative Commons Attribution 4.0 International License.

Read Full License 


\section{Abstract \\ Background}

Extensor mechanism disruption following total knee arthroplasty is a destructive complication with poor outcomes. Presently, limited data exists regarding the direct repair therapy and long-term outcomes. This study was to evaluate the clinical results and complications of direct repair therapy, and compare it with similar studies to determine whether there is a better treatment.

\section{Methods}

During the period of 2008 to 2020, 31 patients underwent direct repair for an extensor mechanism disruption after total knee arthroplasty (15 patellar fractures, 9 patellar tendon disruptions, and 7 quadriceps tendon disruptions). Mean follow-up was 4.9 years. Demographic, operative, and clinical data were collected. The following statistical methods will be employed to analyze the data: descriptive statistics, paired t test, and the Kaplan-Meier method.

\section{Results}

For all 31 patients underwent direct repair for extensor mechanism disruption, 6 patients failed : 2 knees (6\%) of infections and 4 knees (13\%) of re-rupture. ROM was $94.1^{\circ} \pm 15.7^{\circ}$ preoperatively to $73^{\circ} \pm 52^{\circ}$ postoperatively, average extensor lag reduced from $45^{\circ}$ to $20.2^{\circ}$ at follow-up, and the WOMAC and HSS averaged 65 and 72 points. The Kaplan-Meier estimated survivorship with failure for complications as the end point was $81 \%$ ( $95 \%$ confidence interval [95\% Cl], $42.7 \%$ to $73.3 \%$ ) at 12 years.

\section{Conclusion}

Direct repair of the extensor mechanism disruption is not an ideal therapy, it's actually ineffective for the recovery of knee joint function in patients, and will remain severe knee extension lag. No matter which part of the extensor mechanism disruption, direct repair should not be the preferred treatment

\section{Introduction}

Extensor mechanism (EM) disruption after total knee arthroplasty (TKA) is a catastrophic complication. Although the occurrence of this injury is rare, with a reported prevalence between $0.1 \%$ and $3 \% 1,2$, it can lead to significant dysfunction for the patient and very tricky to deal with. The cause of the lesion can be traumatic or atraumatic ${ }^{3,4}$. Traumatic rupture is mostly caused by direct trauma, such as a fall ${ }^{4}$. Atraumatic ruptures may occur in degenerative tendons, and are usually secondary to immunocompromised conditions such as rheumatoid arthritis, systemic lupus erythematosus, diabetes 
mellitus or long-term use of steroids, to an infection, or to iatrogenic causes ${ }^{5,6}$. The extensor mechanism disruption mainly occurred in the quadriceps tendon, patella, and patellar tendon ${ }^{1}$.

Non-operative treatment of these injuries often results in chronic pain, difficulty with ambulation, extensor lag, decreased range of motion (ROM), limb instability, and increased fall risk ${ }^{7}$.Operation must be considered if return to previous level of function and optimal range of motion is desired. There are multiple surgical options for the extensor mechanism disruption, including direct repair (eg, suture or suture Anchor) ${ }^{8}$,reconstruction with allograft (eg, Achilles tendon allograft and whole extensor mechanism allograft), augmentation (eg, semitendinosus tendon) ${ }^{9,10}$, synthetic material (eg, Marlex mesh) ${ }^{11}$.Direct repair and reconstruction of the extensor mechanism present many challenges both to the surgeon as well as the patient, and there is no consensus regarding optimal treatment. There is a lot of reports about reconstruction of the extensor mechanism at present. Nevertheless, there are very limited published reports of direct repair of extensor mechanism disruption in arthroplasty patients.

The primary aim of this study is to present long-term results of direct repair following patellar fracture[ patellar tendon and quadriceps tendon rupture after TKA, and determine whether direct repair for extensor mechanism disruption following TKA have a good outcome. Secondary aim of this study is to compare these results to similar studies to summarize potential advantages and disadvantages.

\section{Material And Methods}

We retrospectively reviewed the medical records of all patients who underwent TKA from 2008 to 2020 were eligible for inclusion in this study. Patients who extensor mechanism disruption after total knee arthroplasty were recognized. Patients with extensor mechanism injuries and limited motion of hip or ankle before surgery were excluded. Sex, age, weight, height of each patient was noted. Radiological data included a weightbearing full length lower extremity antero-posterior radiograph performed with centered patella showing the entire lower limb, a medio-lateral view performed strictly perpendicularly to the superior face of tibial condyles, and an anterio-posterior view performed with strictly centered patella. These radiological data included films taken by the patient before and 3 months after operation. A consecutive series of 31 patients ( 1 men and 30 women; mean age 69 years; age range 57-80 years) were found to have extensor mechanism disruption following TKA. The preoperative diagnosis was osteoarthritis in 30, inflammatory arthritis in one. Patellar fracture was found in 15 patients, quadriceps tendon rupture in 7 patients, and patellar tendon rupture in 9 patients. All the patients were female except 1 male with patellar tendon rupture due to postoperative infection. The median follow-up period was 4.9 years (range 2.0-12 years). The average time from EM disruption to repair was $1.8 \pm 2.1$ months. Except for two patients, the time from injury to repair was 8 months and 5 months respectively, and the rest of the patients were within 3 months. No patients were lost to follow-up. All surgery was performed by a senior surgeon. Patients were clinically assessed pre- and post-operation using ROM, extensor lag, Western Ontario and McMaster Universities (WOMAC), the Hospital for Special Surgery Knee Score (HSS),and radiographic evaluation. 
For patients with extensor mechanism destruction after TKA, we mainly adopted the direct repair. The method of operation was to use two No. 5 non-absorbable sutures running in a locked fashion on the medial and lateral aspect of the quadriceps tendon, then two sutures are passed through the two longitudinal patellar tunnels and sutured to the medial and lateral sides of the patellar tendon(Fig. 1-A,B), followed by suturing the joint capsule[Fig. 1-C区.Finally we tied the patella with a circular suture of titanium cable, then tightened the titanium cable to form the tension band, fixed the titanium cable with cable clamps, and cut off the excessive titanium cableIFig. 1-DQ. This method is mainly aimed at quadriceps tendon fracture combined with patella fracture cases. If only the quadriceps tendon is ruptured, the patella does not need to be encircled with titanium cables. When patellar tendon rupture following a patellar fracture or only patellar tendon rupture, the distal end of the titanium cable needs to pass through the tibial tubercle. The reason for this is to reduce tension on the patellar tendon during postoperative knee motion. In the process of stitching, the suture lines must be continuously tightened to ensure that the EM remains a certain tension and the fractured patella clings closely together. All patients were immobilized with a brace for at least 6 weeks and instructed to stay non-weight bearing, after that they were allowed to start partial weight bearing with passive and active physiotherapy.

\section{Statistical Analysis}

Descriptive statistics are reported as the mean and standard deviation, as the mean and range, or as the number and percentage, as appropriate. Survivorship analysis with failure for complications was calculated with use of the Kaplan-Meier method. Potential risk factors for these events included age, sex, BMI. All statistical tests were 2-sided, the differences in variables were compared using the paired $t$ test, and a p level of $<0.001$ was considered significant. Analysis was performed using SPSS Version 25.0 software (IBM, Armonk, NY, USA).

\section{Results}

Demographic and clinical data are summarized in Table 1 (It's placed at the end of the document text file). Our case series included quadriceps tendon rupture patellar tendon rupture and patellar fracture. For all 31 patients who underwent direct repair for an extensor mechanism disruption, ROM was $94.1^{\circ} \pm 15.7^{\circ}$ preoperatively to $73^{\circ} \pm 52^{\circ}$ postoperatively, average extensor lag reduced from $45^{\circ}$ to $20.2^{\circ}$, and the WOMAC and HSS averaged 64 and 72 points (all $p<0.001$ ). Among these patients, the patient with the best recovery had a postoperative range of motion of $120^{\circ}$, extensor lag of only $5^{\circ}$, HSS score of 95 , and WOMAC score of 8 . However, the patient with the worst recovery had a postoperative range of motion of $66^{\circ}$, extensor lag of $60^{\circ}$, HSS score of 40, and WOMAC score of 85. The Kaplan-Meier estimated survivorship with failure for complications as the end point was $81 \%(95 \%$ confidence interval [95\% Cl], $42.7 \%$ to $73.3 \%$ ) at 12 years (Fig. 2). The complication rate was $19 \%$ : 2 knees (6\%) of infections and 4 knees $(13 \%)$ of re-rupture, and all underwent reoperation. 
Table 1

Demographic and clinical data of 31 patients

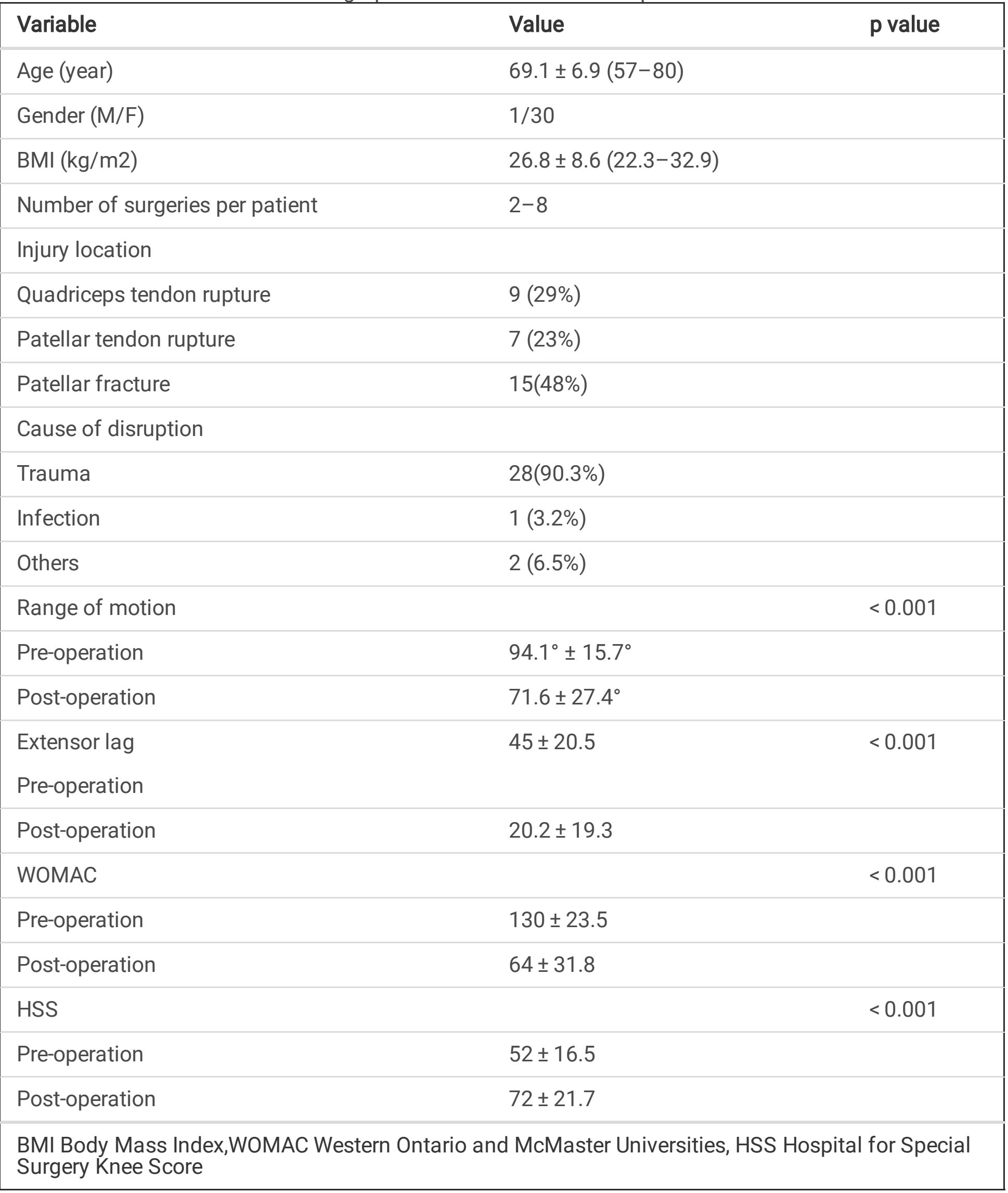


Of the two patients with postoperative infection, one underwent a total of 8 operations due to complications after primary TKA (Fig. 3). This patient underwent primary right TKA because of pigmented vil-lonodular synovitis. After this operation, patient's knee was treated with irrigation and debridement, polyethylene liner exchange due to knee pain and swelling. One year and six months later, the patient's knee joint was painful and swollen again, then the patient was treated with revision total knee arthroplasty, smooth recovery after operation. Unfortunately, half a year later, the patient had a right patellar fracture due to trauma (Fig. 3-A). 20 days after open reduction, internal fixation and titanium cable ring ligation (Fig. 3-B), the patient had black skin necrosis at the surgical incision and was treated with gastrocnemius muscle flap with split-thickness skin graft (Fig. 3-C). Five months after skin flap transplantation, the surface of the right knee joint was ruptured and the sinus appeared (Fig. 3-D). The postoperative knee joint infection was diagnosed, and the synovial fluid culture judged to be staphylococcus aureus infection, then irrigation and debridement, polyethylene liner exchange was performed. During the operation, it was found that the osteoporosis of the patella was serious and the volume of the patella was too small, so the patella was removed and the broken ends of the quadriceps tendon and the patellar tendon were sutured to each other. In the following one year, the patient had three recurrent infections of the right knee joint, two debridement of the knee joint, one puncture of the knee joint cavity and intravenous antibiotic treatment. The infection symptoms of the patient were basically controlled, but the function of the right knee joint was seriously damaged, only slightly bent, and the skin condition on the surface of the knee joint was poor.

Another patient with postoperative infection underwent primary knee arthroplasty because of right knee osteoarthritis, and underwent 4 operations because of postoperative complications after primary TKA. The patient suffered from patellar tendon rupture due to trauma, and was treated with suture anchor repair technique in the other hospital (Fig. 4-A). However, a large amount of exudation occurred in the knee incision after operation, so open irrigation, debridement, liner exchange was performed, but the knee was painful and had poor flexion. A few months later, the patient was treated with open reduction and internal fixation with titanium cable ring (Fig. 4-B). Unfortunately, the titanium cable in the knee joint was broken and the broken titanium cable punctured the skin to form a sinus (Fig. 4-C). To make matters worse, we found Streptococcus aureus in the patient's synovial fluid. After consideration, we performed revision total knee arthroplasty on the patient, and reconstructed the extensor mechanism with Marlex mesh (Fig. 4-D). After operation, the knee joint was fixed with plaster for 6 weeks, gradually increasing the range of motion, and the inflammatory index tended to be normal. For 2 patients with re-rupture after operation, we used semitendinosus as autograft to reconstruct extensor mechanism. After operation, 2 patients achieved a good final outcome, with good range of motion of the knee joint at the latest followup.

\section{Discussion}

The most important findings of the present study were that the result of direct repair after extensor mechanism disruption showed poor outcomes at long-term follow-up. The postoperative patients had low range of motion, accompanied by severe extension lag, and the incidence of postoperative complications 
was high. Previous studies were limited by the relatively small case number as well as only investigating a certain site of extensor mechanism disruption. We provided a more complete and more comprehensive case series. Second, our study showed a low success rate of direct repair and low patient satisfaction compared with other studies. Other treatments shown better postoperative results. We suggest that direct repair should not be the first choice for the treatment of extensor mechanism disruption.

Although extensor mechanism disruption was a rare complication following total knee arthroplasty with incidence ranging from $0.1-3 \%^{1,2}$. Once it happened, it would have a devastating impact on the patients' daily life after operation, and often resulted in frequent falls, difficulty in walking, and pain ${ }^{12,13}$. The treatment of extensor mechanism disruption after TKA had remained a challenge for decades ${ }^{14}$, the ideal method of treatment remained unclear. There have been few recent reports of direct repair after extensor mechanism disruption in arthroplasty patients, except for published in $2005^{15}$ and $2018^{16}$. Our current case series provides an updated report about this challenging complication, including quadriceps tendon rupture $[$ patellar tendon rupture and patellar fracture.

Tracing back to the source, we found two reports about direct repair of extensor mechanism disruption after TKA in earlier years. In 1987, Lynch et al. reported extensor mechanism complications in 281 TKA $^{17}$. Among these complications were eight extensor mechanism disruptions. Of the four patellar tendon ruptures were direct repaired, one re-ruptured at four weeks after the operation, two had residual extensor lags of $18^{\circ}$ and $22^{\circ}$, and 1 became infected. Of the three quadriceps tendon ruptures were repaired primarily, one re-ruptured at six weeks after the operation, two with residual extensors lags of $12^{\circ}$ and $19^{\circ}$, and knee flexion of only $70^{\circ}$. One patella fracture was repaired went on to develop avascular necrosis requiring patellectomy with subsequent post-operative infection and ultimately a knee arthrodesis. In the report of Lynch et al, the failure rate of directly repair of extensor mechanism disruption is $50 \%$, the average residual extensor lag in successful cases was $17.8^{\circ}$, and knee flexion of $70^{\circ}$. In 1989 , Rand et al. reported extensor mechanism complications in 16 TKA, all of which were repaired directly except 3 cases $^{10}$. Of the nine patella tendon ruptures were treated with direct repair, all nine failed with six rerupturing after the operation and three became infected. In the report of Rand et al, the failure rate of directly repair of extensor mechanism disruption is $85 \%$, the average residual extensors lag in successful cases was $1^{\circ}$, and average knee flexion of $81^{\circ}$. While the results from the Lynch et al and the Rand et al. studies showed less extension lag compared to our current study, only slightly floating compared to ROM, but the incidence of postoperative complications was much higher than ours. We studied more patients (31 vs. 8 and 13) which may account for this difference.

Dobbs et al ${ }^{15}$ and Courtney et al $^{16}$ are two rare reports on direct repair after extensor mechanism disruption in recent years. In the report of Dobbs ${ }^{15}, 26$ patients with rupture of quadriceps tendon underwent direct repair. The results of the report are divided into complete rupture and partial rupture of the quadriceps tendon parts. In 10 patients of complete rupture, the rate of postoperative complications was $60 \%$, four of the ten patients had a re-rupture of the quadriceps tendon after operative repair, two patients had a deep periprosthetic infection develop, The active range of motion before a complete tear of 
the quadriceps tendon averaged $2^{\circ}$ (range, $0^{\circ}$ to $8^{\circ}$ ) of extension and $106^{\circ}$ (range, $95^{\circ}$ to $135^{\circ}$ ) of flexion. At the time of the last follow-up, the active range of motion averaged $3^{\circ}$ (range, $0^{\circ}$ to $10^{\circ}$ ) of extension and $101^{\circ}$ (range, $60^{\circ}$ to $125^{\circ}$ ) of flexion. In 16 patients of partial rupture, the rate of postoperative complications was $31 \rrbracket$, five of the sixteen patients had a complication including knee instability, rerupture, intraoperative laceration of the popliteal artery, intraoperative fracture of the femoral condyle, or deep periprosthetic infection. The active range of motion in the sixteen patients before the partial tear of the quadriceps tendon averaged $4^{\circ}$ (range, $0^{\circ}$ to $20^{\circ}$ ) of extension and $110^{\circ}$ (range, $90^{\circ}$ to $130^{\circ}$ ) of flexion. The active range of motion after the operative repair averaged $7^{\circ}$ (range, $0^{\circ}$ to $40^{\circ}$ ) of extension and $104^{\circ}$ (range, $90^{\circ}$ to $130^{\circ}$ ) of flexion. All patients had a mean Knee Society knee score of 74 points and a mean Knee Society function score of 44 points. In the report of Courtney et $\mathrm{al}^{16}, 58$ patients with extensor mechanism disruption underwent direct repair. We analyzed the data of this document about direct repair and come to the conclusion that the reoperation rate was 30区, the mean postoperative extensor lag was $4.15^{\circ}$ \and the mean postoperative flexion was $104.9^{\circ}$ the mean postoperative KSS was 121.6. Although the postoperative range of motion of the patients in this report was high, there was no significant improvement compared with that before operation, and the incidence of complications was still very high.

In our research, the failure rate of direct repair was $19 \%$, the average residual extensors lag in cases was $20.2^{\circ}$, and average knee flexion of $71.6^{\circ}$, the WOMAC and HSS averaged 64 and 72 points. Combined with the above four reports, the postoperative range of motion decreased or had hardly changed compared with that before operation, and the motion is limited. What's more serious was that there was severe knee extension lag, it will seriously affect the knee joint function. High incidence of postoperative complications also greatly increased the failure rate of surgery, and the postoperative scores showed poor recovery. It is far from enough to meet the patients' normal daily life after operation. Judging from these results, the result of direct repair is not satisfactory.

In addition to direct repair, there were other multiple surgical options for extensor mechanism disruption, including reconstruction with allograft, autograft and synthetic material ${ }^{18}$. There was no consensus in the report as to the type of extensor mechanism reconstruction that yields the best results. At present, more and more evidence proved that the therapeutic effect of direct repair was unsatisfactory, which made us have to compare with the results of other treatments, in order to judge whether there was a better treatment.

Allograft augmentation had been considered the gold standard with multiple reports ${ }^{1,19,20}$. In the report of Wise et $\mathrm{al}^{21}$, Sixteen patients with 17 reconstructions (10 patellar tendons, 7 quadriceps tendons) were treated with achilles tendon allograft. All patients underwent evaluation at an average of 45.7 months. After reconstruction, the average extensor lag was $6.6^{\circ}$ and average knee flexion was $105.1^{\circ}$, the extensor $\operatorname{lag}\left(6.6^{\circ}\right.$ vs $\left.20.2^{\circ}\right)$ was significantly lower and the knee flexion $\left(105.1^{\circ}\right.$ vs $\left.71.6^{\circ}\right)$ was significantly higher. In addition to Allograft, we found an up-to-date literature on synthetic material. Besides, monofilament polypropylene mesh has emerged as the preferred treatment ${ }^{11}$. In the report of Buller et al ${ }^{11}, 30$ patients were treated with Marlex Mesh. The failure rate of the operation was $27 \%$. Of the successful extensor 
mechanism reconstructions, extensor lag improved a mean of $34^{\circ}$, with the average final lag measuring $9^{\circ} \pm 8^{\circ}$, the average final active range of motion was $9^{\circ}$ to $107 \pm 15$ degrees of flexion. The extensor lag $\left(9^{\circ}\right.$ $\pm 8^{\circ}$ vs $\left.20.2^{\circ}\right)$ was significantly lower and the knee flexion $\left(107 \pm 15^{\circ}\right.$ vs $\left.71.6^{\circ}\right)$ was significantly higher than ours. Similar results were found in comparison with the literature on autograft ${ }^{22}$, where postoperative range of motion and the extensor lag were better than direct repair.

There were several limitations to this study, including its retrospective design. A limitation of our study was the small number of cases due to the low incidence of extensor mechanism disruption. We present the largest series to our knowledge, including quadriceps tendon rupturelpatellar tendon rupture and patellar fracture. The operative reports were also not consistent in commenting on the quality of the tissues, the characteristics of the rupture, or the patellar fracture pattern.

After comparison with these reports, no matter whether we choose allograft, autograft or synthetic material to reconstruct extensor mechanism, the result of direct repair is not as ideal as that of these treatments. And direct repair had the severe extensor lag after operation, which will have a great impact on the postoperative function of the patients. After the failure of direct repair, these reconstruction methods can also be used as remedial measures. Just like the five patients who failed after operation in our cases, three of them received semitendinosus reconstruction and Marlex mesh reconstruction respectively, and all the patients recovered well after operation.

\section{Conclusion}

Direct repair of extensor mechanism disruption showed unsatisfactory clinical results in this group of patients, and direct repair was actually ineffective for the recovery of knee joint function in patients. More and more new surgical techniques had also shown better clinical results. Therefore, we suggested that direct repair was not the preferred method to treat extensor mechanism disruption, but should boldly choose other reconstruction methods. Our experience of extensor mechanism disruption would be instructive for clinicians to treat extensor mechanism disruption.

\section{Abbreviations}

EM

Extensor mechanism ; TKA:Total knee arthroplasty; BMI:Body Mass Index; WOMAC:Western Ontario and McMaster Universities; HSS:the Hospital for Special Surgery Knee Score; ISR:Insall-Salvati ratio; ROM:Range of motion;

\section{Declarations}

\section{Ethics approval and consent to participate}

This retrospective review study involving human participants was in accordance with the ethical standards of the institutional and national research committee and with the 1964 Helsinki Declaration 
and its later amendments or comparable ethical standards. The study was approved by the medical ethics committee of our hospital. Informed consent Informed consent was obtained from all individual participants included in the study

\section{Consent for publication}

Not applicable.

\section{Availability of data and material}

All data generated or analyzed during this study are included in this published article.

\section{Competing interests}

The authors declare that they have no competing interests.

\section{Funding}

This research did not receive any specific grant from funding agencies in the public, commercial, or notfor-profit sectors.

\section{Author's contributions}

All authors made substantial contributions to conception and design, acquisition of data, or analysis and interpretation of data; took part in drafting the article or revising it critically for important intellectual content; gave final approval of the version to be published; and agree to be accountable for all aspects of the work.

\section{Acknowledgements}

The authors would like to thank all staff from the participating departments and clinics.

\section{References}

1. Bates M, Springer B. Extensor mechanism disruption after total knee arthroplasty. J Am Acad Orthop Surg. 2015;23(2):95-106.

2. Maffulli N, Spiezia F, La Verde L, Rosa M, Franceschi F. The Management of Extensor Mechanism Disruption After Total Knee Arthroplasty: A Systematic Review. Sports Med Arthrosc. 2017;25(1):4150.

3. Cottino U, Abdel M, Hanssen A. Chronic extensor mechanism insufficiency in total knee arthroplasty (TKA). Curr Rev Musculoskelet Med. 2015;8(4):368-72.

4. Bonnin M, Lustig S, Huten D. Extensor tendon ruptures after total knee arthroplasty. Orthop Traumatol Surg Res. 2016;102:21-31. 
5. Goldstein Z, Yi P, Haughom B, Hellman M, Levine B. Bilateral extensor mechanism disruption after total knee arthroplasty in two morbidly obese patients. Orthopedics. 2015;38(5):e443-6.

6. Seng C, Lim Y, Pang H. Spontaneous disruption of the bilateral knee extensor mechanism: a report of two cases. J Orthop Surg (Hong Kong). 2015;23(2):262-6.

7. Lim C, Amanatullah D, Huddleston J, Harris A, Hwang K, Maloney W, Goodman S. Reconstruction of Disrupted Extensor Mechanism After Total Knee Arthroplasty. J Arthroplasty. 2015;32(10):3134-40.

8. Kim T, Kamath A, Israelite $C$. Suture anchor repair of quadriceps tendon rupture after total knee arthroplasty. J Arthroplasty. 2011;26(5):817-20.

9. Cadambi A, Engh G. Use of a semitendinosus tendon autogenous graft for rupture of the patellar ligament after total knee arthroplasty. A report of seven cases. J Bone Joint Surg. 1992; Am 74(7):974-979.

10. Rand J, Morrey B, Bryan R. Patellar tendon rupture after total knee arthroplasty. Clin Orthop Relat Res. 1989; (244):233-238.

11. Buller L, Warth L, Deckard E, Meneghini R. Extensor Mechanism Reconstruction Using Marlex Mesh: Is Postoperative Casting Mandatory? J Arthroplasty. 2020;35(12):3747-53.

12. Barrack R, Stanley $T$, Allen Butler R. Treating extensor mechanism disruption after total knee arthroplasty. Clin Orthop Relat Res. 2003;(416):98-104.

13. Emerson R, Head W, Malinin T. Reconstruction of patellar tendon rupture after total knee arthroplasty with an extensor mechanism allograft. Clin Orthop Relat Res. 1990;(260):154-161.

14. Nam D, Abdel M, Cross M, LaMont L, Reinhardt K, McArthur B, Mayman D, Hanssen A, Sculco T. The management of extensor mechanism complications in total knee arthroplasty. AAOS exhibit selection. J Bone Joint Surg Am. 2014;96(6):e47.

15. Dobbs R, Hanssen A, Lewallen D, Pagnano M. Quadriceps tendon rupture after total knee arthroplasty. Prevalence, complications, and outcomes. J Bone Joint Surg Am. 2015;87(1):37-45.

16. Courtney P, Edmiston T, Pflederer C, Levine B, Gerlinger T. Is There Any Role for Direct Repair of Extensor Mechanism Disruption Following Total Knee Arthroplasty? J Arthroplasty. 2018;33:244-8.

17. Lynch A, Rorabeck C, Bourne R. Extensor mechanism complications following total knee arthroplasty. J Arthroplasty. 1987;2(2):135-40.

18. Papalia R, Vasta S, D'Adamio S, Albo E, Maffulli N, Denaro V. Complications involving the extensor mechanism after total knee arthroplasty. Knee Surg Sports Traumatol Arthrosc. 2015;23(12):350115.

19. Springer B, Della Valle C. Extensor mechanism allograft reconstruction after total knee arthroplasty. J Arthroplasty. 2008;23:35-8.

20. Diaz-Ledezma C, Orozco F, Delasotta L, Lichstein P, Post Z, Ong A. Extensor mechanism reconstruction with achilles tendon allograft in TKA: results of an abbreviate rehabilitation protocol. J Arthroplasty. 2014;29(6):1211-5. 
21. Wise B, Erens G, Pour A, Bradbury T, Roberson J. Long-term results of extensor mechanism reconstruction using Achilles tendon allograft after total knee arthroplasty. Int Orthop. 2018;42(10):2367-73.

22. Lamberti A, Balato G, Summa P, Rajgopal A, Vasdev A, Baldini A. Surgical options for chronic patellar tendon rupture in total knee arthroplasty. Knee Surg Sports Traumatol Arthrosc. 2018;26(5):1429-35.

\section{Figures}

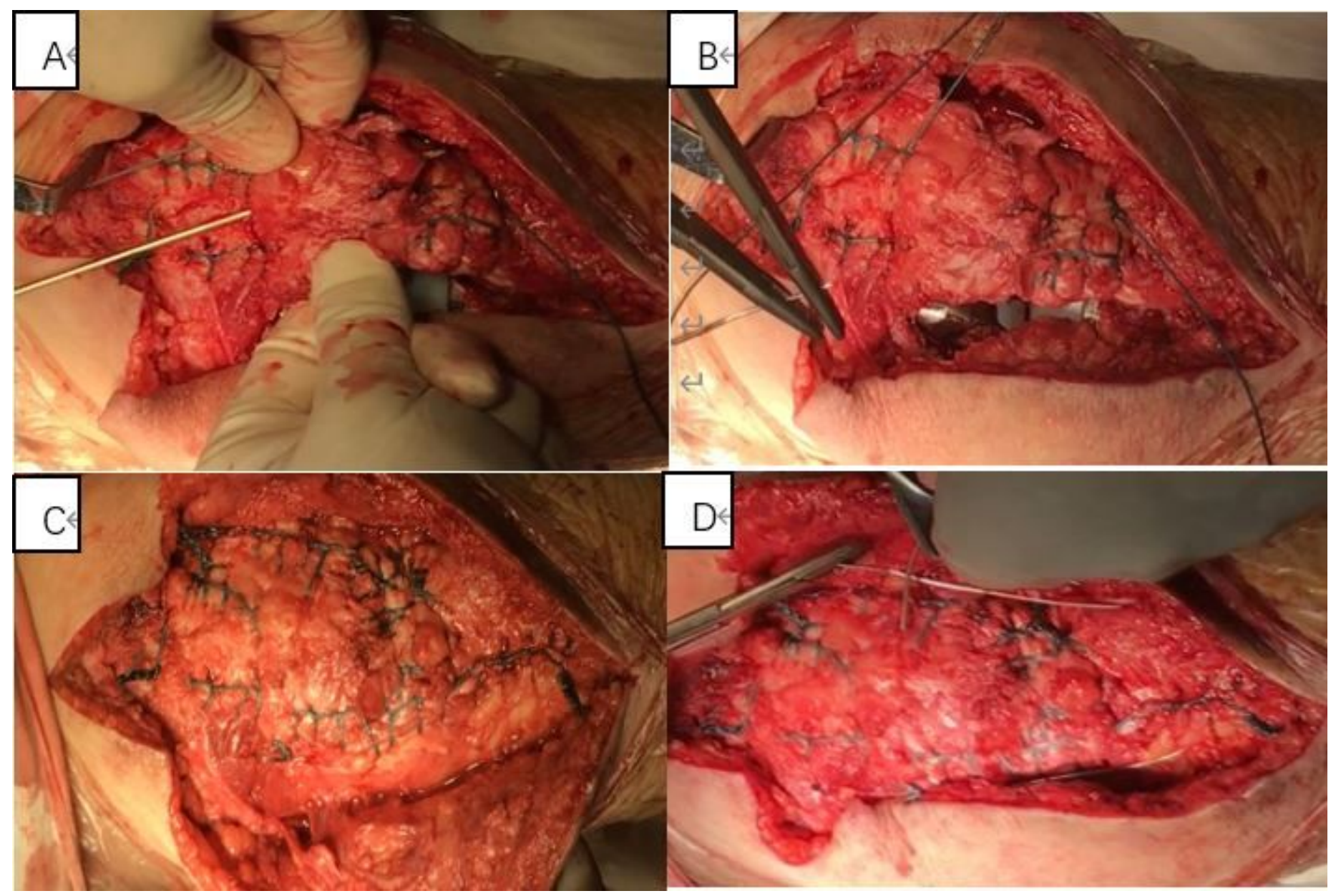

\section{Figure 1}

The first method of operation was to use No. 5 non-absorbable suture for end-to-end suture, and the second method of operation was to use two No. 5 non-absorbable sutures running in a locked fashion on the medial and lateral aspect of the quadriceps tendon, and sutured through two bone channels drilled longitudinally from the patella to both sides of the patellar tendon (Fig. 1-A囚B囚. Then sutured EM to both tissues and closed the joint capsulखFig. 1-C囚. Finally tied the patella with a circular suture of titanium cable, then tightened the titanium cable to form the tension band, fixed the titanium cable with cable clamps, and cut off the excessive titanium cable®Fig. 1-D囚 


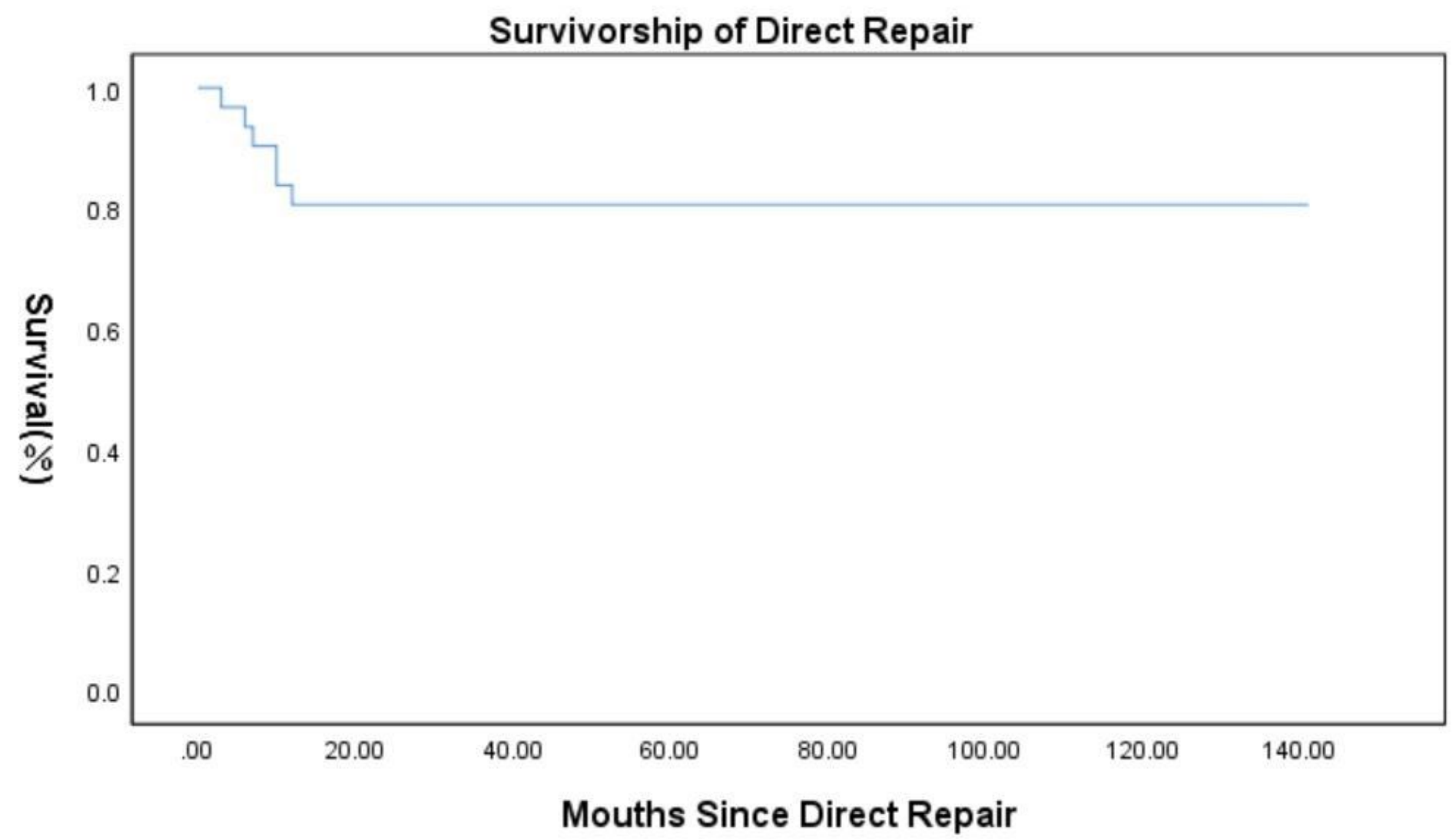

Figure 2

Kaplan-Meier curves showing survivorship of direct repair for patients. 

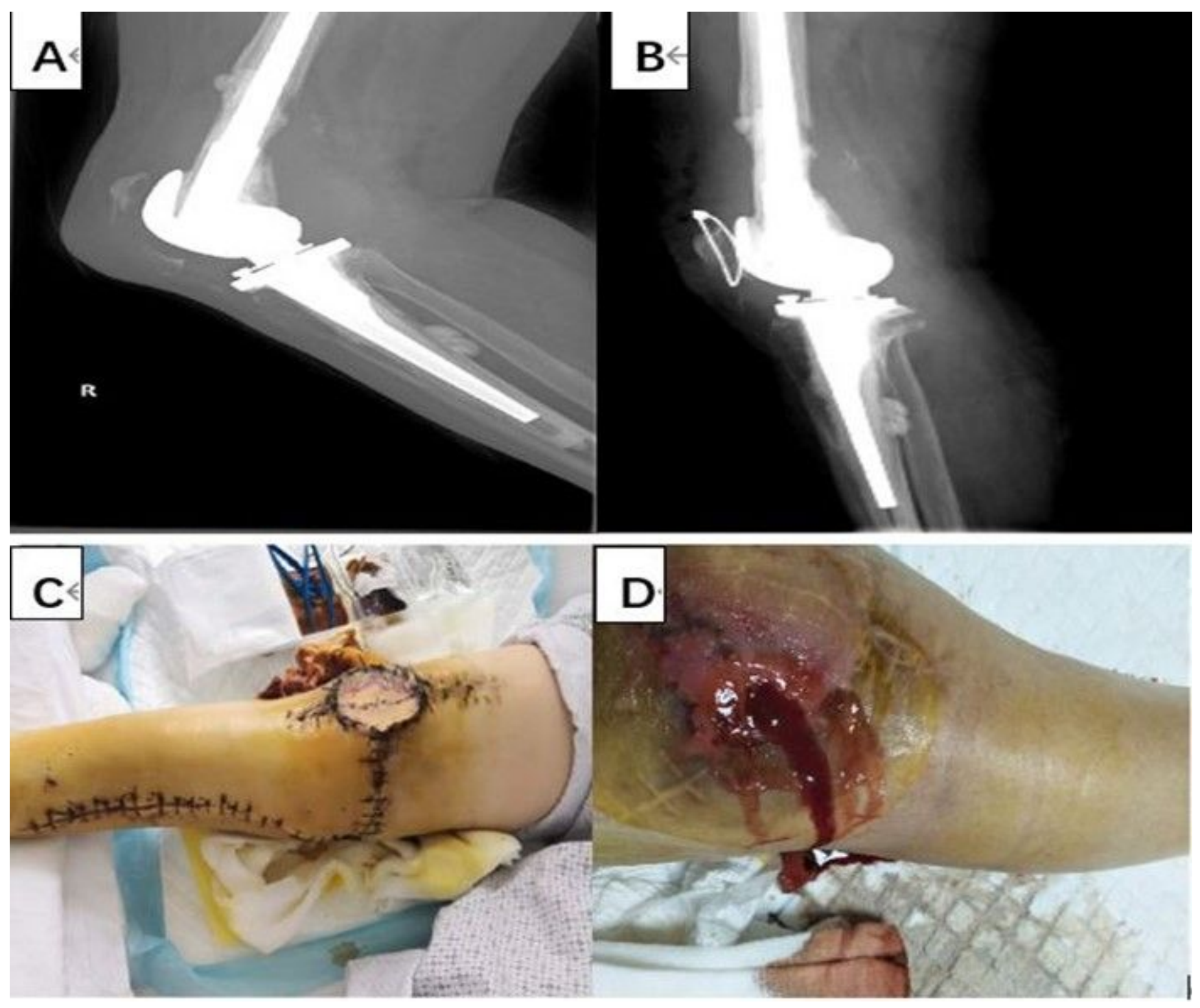

Figure 3

X-rays (A) of patient with patella fracture due to trauma. X-ray film(B) after direct repair of patellar fracture. The patient $(C)$ was treated with gastrocnemius muscle flap with split-thickness skin graft because of skin necrosis of surgical incision. Sinus(D) appears on the skin surface of the right knee joint. 

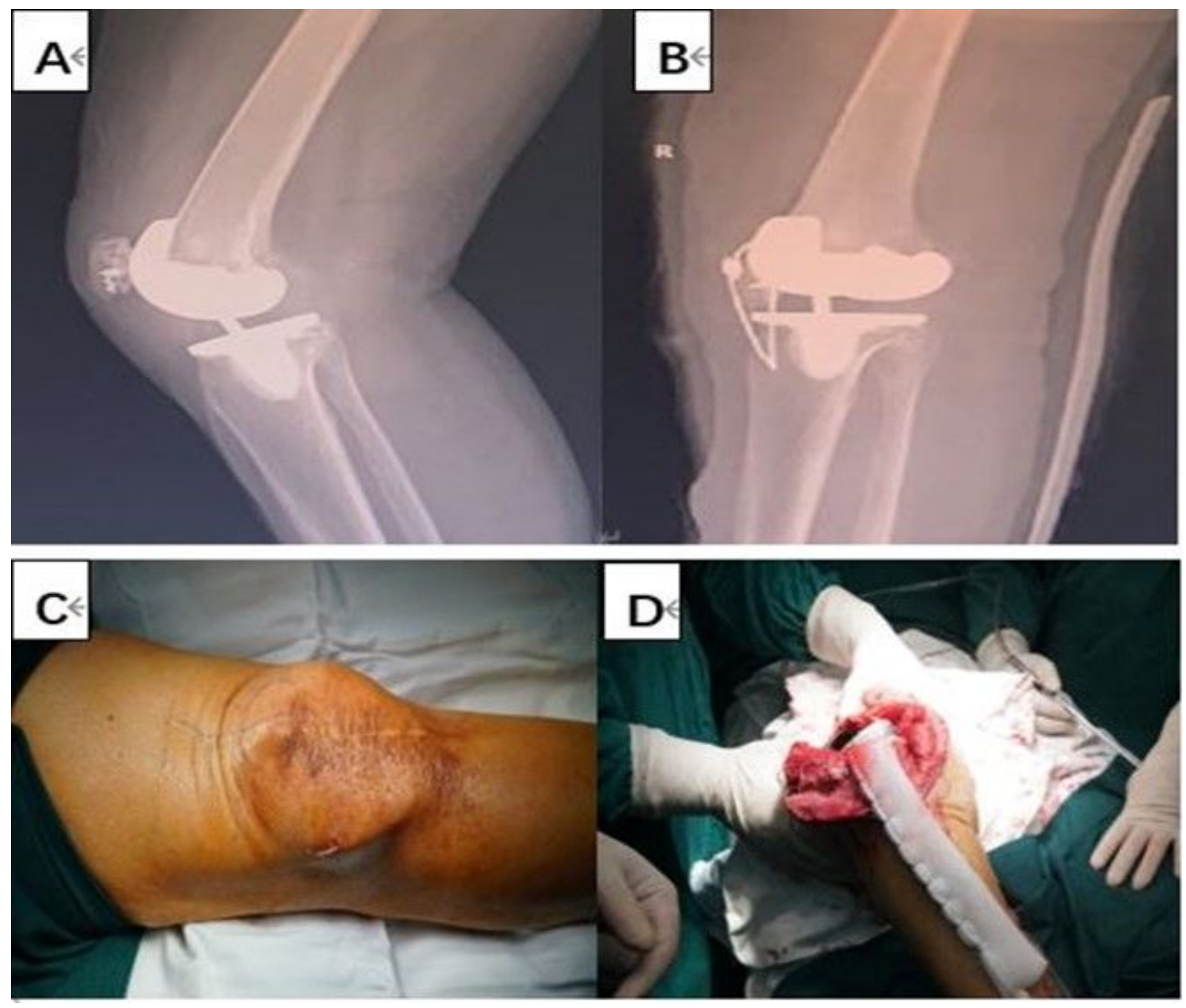

\section{Figure 4}

The X-ray $(A)$ of the patient after suture anchoring treatment in the other hospital. The X-ray $(B)$ after direct repair of patellar tendon fracture. The titanium cable(C) in the knee joint was broken and the broken titanium cable punctured the skin to form a sinus. The patient (D)was treated with Marlex mesh. 